\title{
Estructura de planeación orientada a fortalecer el trabajo de los cuerpos académicos de una facultad de ingeniería
}

\section{Planning structure aimed at strengthening the work of the academic bodies of an engineering faculty}

DIMAS-RANGEL, María Isabel*†, TORRES-BUGDUD, Arturo, PALOMARES-RUIZ, María Blanca Elizabeth y SORDIA-SALINAS, César

Universidad Autónoma de Nuevo León, Facultad de Ingeniería Mecánica y Eléctrica. Pedro de Alba SN, Niños Héroes, Ciudad Universitaria, San Nicolás de los Garza, N.L.

ID $1^{\text {er }}$ Autor: María Isabel, Dimas-Rangel / ORC ID: 0000-0001-9450-8080, CVU CONACYT ID: 374045

ID $1{ }^{\text {er }}$ Coautor: Arturo, Torres-Bugdud / ORC ID: 0000-0003-2214-9394, CVU CONACYT ID: 216332

ID $2^{\text {do }}$ Coautor: María Blanca Elizabeth, Palomares-Ruiz / ORC ID: 0000-0002-4079-6969, CVU CONACYT ID: 339594

ID $3^{\text {er }}$ Coautor: César, Sordia-Salinas / ORC ID: 0000-0003-2186-1080, CVU CONACYT ID: 339888

DOI: $10.35429 /$ JOTE.2019.8.3.23.31

Recibido 10 de Abril, 2019; Aceptado 17 de Junio, 2019

Resumen

El compromiso que la Institución de Educación Superior en México tiene con el país es importante, pues su contribución en la formación de profesionistas de calidad es un factor trascendente que impacta en la sociedad. Considerando lo anterior en el presente trabajo investigativo se reflexiona en relación a la trascendencia de fortalecer el trabajo colegiado en beneficio de los Programas Educativos de ingeniería, para así favorecer la formación ingenieril mediante la labor que desarrollan los académicos expertos en áreas educativas afines. Debido a la necesidad de promover el desarrollo investigativo de la planta de profesores en beneficio de la docencia, se promueve el fortalecimiento del perfil del profesor a través de estrategias que lo motivan a desarrollar otras actividades que favorecen en su desarrollo profesional e impactan positivamente en los estudiantes. La propuesta aquí presentada tiene como base estratégica promover de manera sistémica una estructura administrativa de planeación que integra los factores que son importantes para fortalecer el trabajo colaborativo de los profesores, la cual posibilita visualizar la trayectoria de desarrollo que pueden tener los miembros de un cuerpo colegiado. Dicha planeación se diseñó en base a un análisis documental, se implementa mediante el método sistémico-estructural, se analiza utilizando estadística descriptiva y se mejora mediante análisis de los resultados obtenidos. La sistematización de estas estrategias de gestión académicoadministrativa favorecen en la evolución de estos equipos de trabajo, incrementando en un 57\% la cantidad Cuerpos Académicos Consolidados y en un 64\% Cuerpos Académicos en Consolidación, lo que contribuye en el fortalecimiento de la Capacidad Académica de la Facultad de Ingeniería, e impactando positivamente en formación integral de los futuros ingenieros.

Cuerpos académicos, planeación operativa, capacidad académica
Abstract

The commitment that the Higher Education Institution in Mexico has with the country is important, since its contribution in the formation of quality professionals is a transcendent factor that impacts society. Considering the foregoing, this research work reflects on the importance of strengthening collegial work for the benefit of engineering Educational Programs, in order to favor engineering education through the work developed by academic experts in related educational areas. Due to the need to promote the research development of the teaching staff, the strengthening of the teacher's profile is promoted through strategies that motivate him to develop other activities that favor his professional development and have a positive impact on the students. The strategic basis of the proposal presented here is to promote, in a systemic manner, an administrative planning structure that integrates the factors that are important for strengthening the collaborative work of teachers, which makes it possible to visualize the development trajectory that members of a collegiate body may have. This planning was designed on the basis of a documentary analysis, it is implemented through the systemic-structural method, it is analyzed using descriptive statistics and it is improved through analysis of the results obtained. The systematization of these strategies of academicadministrative management favor in the evolution of these work teams, increasing in 57\% the amount of Consolidated Academic Bodies and in $64 \%$ of Academic Bodies in Consolidation, which contributes in the strengthening of the Academic Capacity of the Faculty of Engineering, and positively impacting in integral formation of the future engineers.

Academic bodies, Academic bodies planning, Academic ability

Citación: DIMAS-RANGEL, María Isabel, TORRES-BUGDUD, Arturo, PALOMARES-RUIZ, María Blanca Elizabeth y SORDIA-SALINAS, César. Estructura de planeación orientada a fortalecer el trabajo de los cuerpos académicos de una facultad de ingeniería. Revista de Educación Técnica. 2019. 3-8: 23-31.

*Correspondencia al Autor (Correo Electrónico: maria.dimasrn@uanl.edu.mx)

$\dagger$ Investigador contribuyendo como primer autor. 


\section{Introducción}

En la actualidad, se destaca la importancia de la educación como un instrumento que contribuye al crecimiento de un país, sin duda esto es cierto ya que como menciona la UNESCO "la educación de calidad propicia el desarrollo de las competencias, los valores y las actitudes que permiten a los ciudadanos llevar vidas saludables y plenas, tomar decisiones con conocimiento de causa y responder a los desafíos locales y mundiales mediante la Educación para el Desarrollo Sostenible (ESD) y la Educación para la Ciudadanía Mundial (ECM)". (UNESCO, 2016). Se identifica que lo antes mencionado es una directriz que favorece a la nación en general y particularmente a sus ciudadanos, y es debido a esto que la Organización para la Cooperación y el Desarrollo Económicos (OCDE) promueve tres prioridades clave (Ramos, 2019):

1. Asegurar que la inversión en educación superior sea adecuada para garantizar la calidad.

2. Ofrecer a los estudiantes mejores garantías de la calidad de la educación que reciben.

3. Una mejor correspondencia entre los conocimientos y las competencias que los estudiantes adquieren y las necesidades del mundo laboral.

Y para contribuir a estas prioridades el contexto mexicano a través de la Secretaria de Educación Pública, mediante su Plan de Desarrollo, destaca la participación de la Institución Educativa (IE) en el progreso de los individuos de su país, en este trabajo investigativo, el alcance considerado son las Instituciones de Educación Superior (IES), ya que estas posibilitan la profesionalización de individuos al contribuir con su desarrollo e integración al campo laboral.
La participación de la institución educativa para el logro de lo anteriormente mencionado requiere de sistematizar las buenas prácticas que se desarrollan en el contexto educativo así mismo de un análisis objetivo que permita identificar cuáles son las necesidades a atender en la formación de los estudiantes y es con este propósito que la propuesta aquí presentada se orienta a contar con una estrategia que coadyuva a fortalecer los programas académicos que se imparten a nivel licenciatura en una institución de educación superior mediante el trabajo investigativo, docente, tutorial y de gestión que realizan sus profesores. Ya que la realización de estas acciones vinculadas al ámbito escolar favorecen en el desarrollo de los individuos en formación, en beneficio de la sociedad. Con la intención de que las acciones académicas mencionadas contribuyan al logro de los objetivos institucionales en el contexto de estudio, se promueve el ejercicio de planeación ya que se considera como un elemento trascendente para la implementación de dichas actividades, las cuales están orientadas a contribuir a la misión de la Institución Educativa (IE).

Este ejercicio de planeación proyecta el trabajo de los académicos organizado en diferentes líneas educativas que favorezcan en la realización de las diversas actividades académicas con que participa el docente como parte de un grupo de trabajo orientado hacia la investigación en beneficio de los programas educativos y que en el contexto mexicano se le denomina Cuerpos Académicos (CA) a los "Grupos de profesores/as de tiempo completo que comparten una o varias líneas de generación de conocimiento, investigación aplicada o desarrollo tecnológico e innovación en temas disciplinares o multidisciplinares, un conjunto de objetivos y metas académicas. Adicionalmente atienden los programas educativos afines a su especialidad en varios tipos" (Secretaría de Educación Pública, 2019).

Estos grupos de trabajo se integran por profesores de áreas afines con la finalidad de cultivar Líneas de Generación y Aplicación del Conocimiento (LGAC) que contribuyen al fortalecimiento de los Programas Educativos de la institución, estas líneas tienen como propósito establecer investigaciones o estudios en temas disciplinares o multidisciplinares. (Secretaría de Educación Pública, 2019) 
El profesorado que integra estos cuerpos colegiados representa un valor importante para los programas educativos, pues su labor es favorable para el enriquecimiento de los mismos, a su vez los nutre con el trabajo investigativo y en paralelo contribuye a la formación del personal docente en diferentes direcciones, la propuesta aquí presentada muestra una estructura sistémica que al planificar aporta en forma sustantiva a los CA y por consiguiente a su desarrollo, así como a su transición, en una Facultad de ingeniería considerando los elementos de influencia de este contexto educativo. Estos grupos de trabajo se formalizan en la institución a partir de su momento de creación, ante un organismo reconocido a nivel nacional adscrito a la Secretaria de Educación Pública mediante la Dirección de Superación Académica, la cual tiene como propósito "Contribuir para que el personal docente, técnico docente y personal con funciones de dirección, de supervisión, de asesoría técnico pedagógica y cuerpos académicos accedan y/o concluyan programas de formación, actualización académica, capacitación y/o proyectos de investigación que les permita fortalecer el perfil para el desempeño de sus funciones." (Secretaría de Educación Pública, 2019); esto en el marco del Programa para el Desarrollo Profesional Docente, para el Tipo Superior (PRODEP), adoptando las reglas de operación que se establezcan para su integración clasificándolos en tres niveles los cuales se muestran a continuación en la Figura 1:

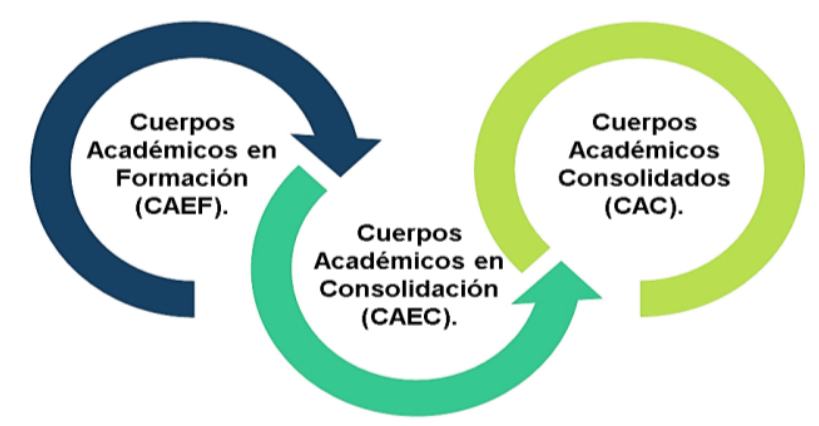

Figura 1 Evolución de los Cuerpos Académicos

Es importante hacer mención, que dentro de la clasificación de los Cuerpos Académicos existen 3 niveles, el primer nivel es Cuerpo Académico en Formación, el segundo es Cuerpo Académico en Consolidación y el tercero es el Cuerpo Académico Consolidado; cada uno específica e implementa las condiciones de los docentes al estar integrado a un grupo de trabajo.
Estos estadios reflejan la evolución de los Cuerpos Académicos a partir de su creación al integrarse como grupos de investigación, se declaran en formación, transitando paulatinamente en base a su desarrollo hacia los siguientes niveles En Consolidación y Consolidado. Alcanzar estos niveles de madurez en los grupos colegiados requiere de una planificación de las actividades académicas afín de obtener resultados positivos que fortalezcan el desarrollo de los profesores. La estructura de planeación aquí presentada posibilita un ejercicio de reflexión orientado hacia la mejora, estableciendo metas orientadas a obtener logros constantes en diferentes categorías que conducen a su evolución y consolidación.

Para la realización de este ejercicio se considera como eje principal dar a conocer, la importancia de la planeación táctica y operativa en los Cuerpos Académicos formados dentro de una Facultad de ingeniería, así como también los elementos a considerar para analizar sus puntos de desarrollo. Tomando como referencia las aportaciones de Münch Galindo, se contextualiza al campo de investigación considerando que la planeación táctica se refiere a los planes específicos, que se elaboran en cada uno de los departamentos o áreas de la organización, enfocándose a un área determinada, pueden ser a mediano y/o a corto plazo; mientras que la operativa es a corto plazo, se diseña y se rige de acuerdo con la planeación táctica, la cual se realiza en diferentes apartados.

Su función consiste en la formulación de estrategias y acciones que contribuyen a la obtención de resultados a mediano plazo que debe cumplir el grupo académico para alcanzar la meta final. (Münch Galindo, 2008) En el contexto de estudio se considera la realización de un ejercicio de planeación clasificado en tres etapas, en primera instancia la realización de un plan estratégico el cual es conocido como Plan de Desarrollo, este establece la visión de la Facultad y sus estrategias para alcanzarlas posteriormente se considera un Plan Táctico el cual se orienta al desarrollo de sus Programas Educativos (PE) y Cuerpos Académicos y finalmente el Plan Operativo el cual se desarrolla para cristalizar las acciones consideradas. 


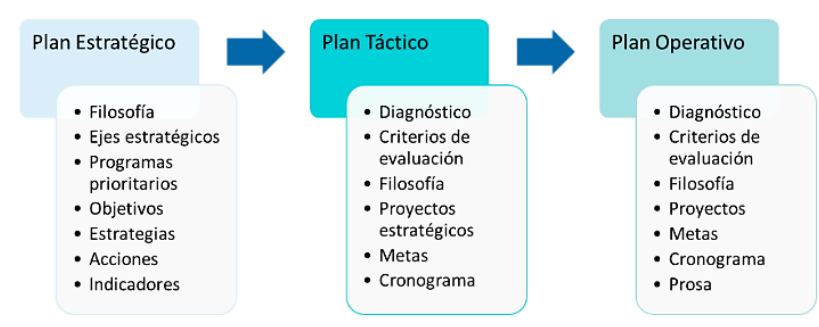

Figura 2 Tipos de Planeación

La anterior Figura muestra el alcance de los elementos considerados para realizar el ejercicio de planeación.

\section{Metodología}

En este apartado se muestra los métodos utilizados para la realización de este trabajo investigativo, el cual se construyó a partir de la utilización de métodos científicos y empíricos que permitieron fundamentar las acciones realizadas. Las bases metodológicas utilizadas fueron:

\section{- Método Análisis Documental}

Este método de análisis, mediante el cual por un proceso intelectual se extraen ideas del documento para representarlo y facilitar el acceso a la consulta del documento original.

(Liniers, 2004)

\section{- Método Analítico}

Este método fue empleado para llevar a cabo el trabajo de investigación documental, tomando como referente a Gutiérrez Sáenz y Sánchez González se procedió a revisar en forma ordenada todos los datos necesarios para la investigación y como ellos lo puntualizan "distinguir las partes de un todo y procede a la revisión ordenada de cada uno de los elementos por separado" (Gutiérrez Sáenz \& Sánchez González, 1990)

\section{- Método sintético}

Este método destacó durante el análisis y síntesis de la información recopilada, lo que permitió ir estructurando las ideas.

\section{- Método inductivo}

El diseño de la estructura de planeación fue resultado de la aplicación de este método ya que es útil a partir del análisis de hechos singulares, que pretende establecer leyes o normas.

(Maya, 2014)

En base al contenido revisado previamente se plantea una hipótesis afín de proponer una estructura sistémica que posibilite el desarrollo de estos grupos colegiados.

\section{Hipótesis:}

$\mathrm{Si}$ se diseña una estructura de planeación orientada a la transición de los cuerpos académicos se podrá contribuir al desarrollo y evolución de los mismos en beneficio de los programas educativos.

\section{Desarrollo}

\section{Planeación de Cuerpos Académicos (CA)}

El contexto de estudio es una Facultad de ingeniería y manifiesta en su misión su compromiso de la formación centrada en el aprendizaje y basado en competencias de ingenieros $\mathrm{e}$ investigadores capaces de desempeñarse eficientemente en la sociedad del conocimiento, que aplican principios y valores universitarios y se comprometen con el desarrollo sustentable, económico, científico, tecnológico y cultural de la humanidad. (Facultad de Ingeniería Mecánica y Eléctrica, 2018)

Esta escuela de ingeniería organiza sus acciones hacia el cumplimiento de la Visión 2020, a través del establecimiento de un Plan de Desarrollo que busca el fortalecimiento de su capacidad y competitividad académica, proponiendo estrategias para el alcance de las metas institucionales a fin de obtener el reconocimiento como una Facultad de ingeniería pública con un alto prestigio mundial. 
El cumplimiento de la Planeación Estratégica se reconoce como el proceso que sirve para formular y ejecutar las estrategias de la organización, con la finalidad de insertarla según su misión en el contexto que se encuentra (Chiavenato, 2016) para el campo de estudio este tipo de planeación se identifica como Plan de Desarrollo, en el contexto de estudio se logra a través de la planeación de las actividades de cada área académico-administrativa que conforma a la Facultad, la cual se encuentra integrada por los Programas Educativos, los Cuerpos Académicos y las áreas administrativas. Dicha planeación consentirá la adecuada toma de decisiones ( Facultad de Ingeniería Mecánica y Eléctrica, 2012).

Como parte de la actividad académica que se realiza al interior de la escuela, se considera a los Cuerpos Académicos como un grupo de trabajo que en base a su labor investigativa, contribuye a la consolidación de los Programas Educativos ofertados en la Facultad, a través del fortalecimiento de la capacidad y competitividad académica, por lo que deben realizar una Planeación Táctica, que define metas a mediano plazo alineadas al Plan de Desarrollo de la Institución, y una Planeación Operativa anual en el que se establecen estrategias y acciones que dan consecución a las metas anuales de su Planeación Táctica.

\section{Planeación Táctica}

Considerando el texto de... identifica la Planeación Táctica como planes específicos que se elaboran en cada una de las áreas afín de contribuir al Plan Estratégico. (Münch Galindo, 2008). Actualmente la Facultad cuenta con un instrumento electrónico que permite la contribución del Cuerpo Académico para el logro de las metas institucionales del Plan de Desarrollo de la Facultad, así como la transición de grado de consolidación del CA, llamado Planeación Táctica (PT). Dicho documento se encuentra estructurado con los siguientes elementos:

a) Análisis de la situación actual.

b) Marco axiológico.

c) Estructura del Plan de Desarrollo del Cuerpo Académico.

d) Indicadores del Plan de Desarrollo

ISSN-2523-2460

ECORFAN $^{\circledR}$ Todos los derechos reservados

\section{Análisis de la situación actual}

Se realiza un autoestudio identificando las fortalezas y debilidades en base a los grados de consolidación del PRODEP en el que se encuentre el CA en el momento de análisis, así como del grado al que se desea transitar, proponiendo acciones para afianzarlas $\mathrm{o}$ superarlas.

Mediante el uso de un sistema electrónico, se puede clasificar el estado actual del Cuerpo Académico según su grado de consolidación según el marco de referencia mencionado con anterioridad. Los grados de consolidación de Cuerpos Académicos son:

- Cuerpo Académico en Formación (CAEF).

- Cuerpo Académico en Consolidación (CAEC).

- Cuerpo Académico Consolidado (CAC).

En la Figura 3 se muestra la caracterización de cada uno de ellos.

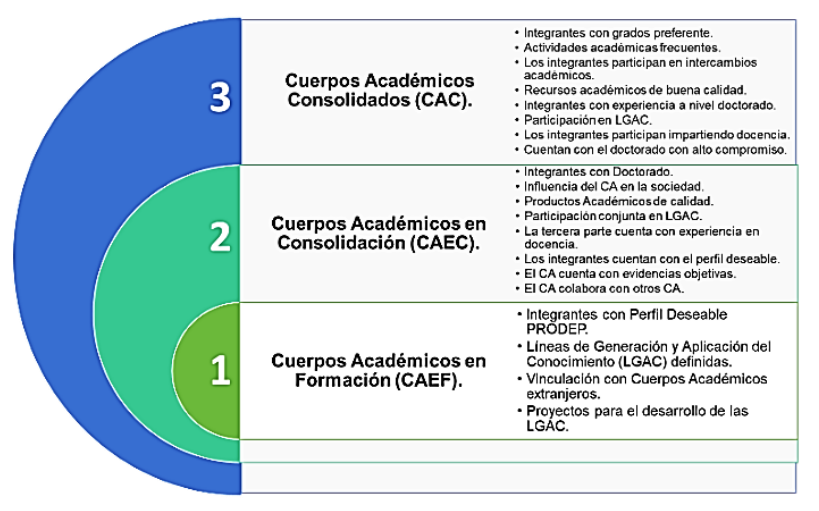

Figura 3 Cuerpos Académicos (PRODEP 2018)

Fuente: elaboración propia

Cada grado cuenta con ciertas características que deben ser atendidas para obtener su reconocimiento por el PRODEP

Otro elemento importante a considerar, es el contexto institucional el cual se documenta a través del siguiente punto:

\section{Marco axiológico}

En este apartado, el CA determina los principios con los que se conduce para el óptimo desarrollo del mismo. Para la construcción de este es importante definir lo siguiente: 
Misión: en ella se establece el propósito de la existencia del CA, así como la actividad clave del mismo que conducirá al logro de su Visión.

Visión: es la percepción a futuro que se espera lograr sobre el desarrollo del CA, la cual debe definirse a un nivel alcanzable y realista.

Valores: Se encuentran definidos en el Plan de Desarrollo de la Facultad. Promueven la identidad y pertenencia del CA a la institución.

Posteriormente se proyecta una estructura que permite planificar las estrategias que contribuirán a la evolución de estos grupos colegiados mediante la generación del "punto c".

\section{Estructura del Plan de Desarrollo del Cuerpo Académico}

Al tener en claro la dirección y objetivo del CA y los principios que regirán su quehacer para la obtención del reconocimiento de alta calidad del PRODEP, se debe estructurar un proyecto para la transición de grado de consolidación del CA.

siguientes elementos:

Objetivo general: es el propósito fundamental, señala el resultado que se espera obtener al finalizar el proyecto. Se encuentra conformado por diferentes objetivos.

Objetivos específicos: son los resultados del logro de las metas y que en conjunto contribuirán al cumplimiento del objetivo general.

Metas: es el resultado de la implementación de estrategias. Deben definirse de manera clara, que sus resultados puedan ser medibles y que se encuentren al alcance del Cuerpo Académico.

Estrategias: muestran el fin al que llegan una serie de acciones. Son la consecución de las metas del proyecto.
Acciones: Estas se definen en el apartado a) Análisis de la situación actual. Es la operación específica que se llevará a cabo para el logro de las estrategias.

Recursos: son los elementos que se utilizarán durante el desarrollo de las acciones del proyecto. Estos pueden ser de tipo económico, material, recurso humano o infraestructura.

Otro aspecto importante a considerar es la medición de logro, el cual se monitorea mediante el cumplimiento de indicadores que permiten observar el desarrollo del cuerpo colegiado.

\section{Indicadores del Plan de Desarrollo}

En base al proyecto antes establecido, habrá que desarrollar el cronograma anual para llevar a cabo las metas que se decidieron en el proyecto y que dirijan al CA a la transición de grado de consolidación que se desea. Durante el desarrollo de las metas establecidas en este cronograma, se programa una evaluación de medio término del Cuerpo Académico.

Este ejercicio de planeación táctica, proyecta las estrategias a realizar a mediano plazo y es necesario complementar este plan con una Planeación Operativa que posibilita la especificidad de las acciones y el seguimiento a las mismas.

\section{Planeación Operativa}

La Planeación Operativa (PO) establece estrategias y acciones que se desean cumplir anualmente y que consolidan la Planeación Táctica del CA. El ejercicio de Planeación Operativa está estructurado en 5etapas que son:

a) Diagnóstico.

b) Filosofía de Calidad.

c) Proyecto.

d) Cronograma.

e) Desarrollo en prosa. 
Es un ejercicio muy similar a la elaboración de la Planeación Táctica del Cuerpo Académico, sin embargo, a continuación, se explica detalladamente las diferencias.

\section{Diagnóstico}

Se analiza la situación actual del Cuerpo Académico considerando 6 indicadores categorizados en base al quehacer institucional, así como también el modelo de evaluación del CONACYT (Consejo Nacional de Ciencia y Tecnología), que son:

Estructura del programa: se analizan todos los indicadores que conforman la competitividad académica para asegurar la calidad y pertinencia de los Programas Educativos ofertados por la Facultad.

Estudiantes: esta categoría incluye todos los rubros relacionados a la adecuada formación integral de la comunidad estudiantil de la Facultad.

Personal Académico: comprende los indicadores relacionados a la capacidad académica afín de proyectar las oportunidades de desarrollo de los profesores. Este ejercicio considera también como insumo importante las reglas de operación proporcionadas por el PRODEP con el propósito de dirigir las acciones para que el personal académico mantenga su reconocimiento de perfil deseable que otorga el PRODEP.

Infraestructura: dentro de esta categoría se incluyen los muebles, bienes inmuebles, material y programación de mantenimiento del equipamiento para el desarrollo de las actividades del Cuerpo Académico.

Productividad Académica: esta categoría está relacionada a todo el material científico y de propiedad intelectual que se genera del quehacer del Cuerpo Académico.

Cooperación con otros actores: son todos los vínculos que permiten la creación de redes de colaboración entre instituciones académicas, organizaciones y/o cuerpos académicos.

\section{Filosofía de Calidad}

De igual forma que la Planeación Táctica, se establece una Misión y Visión del Cuerpo Académico. En esta parte de la PO, se toma en cuenta la Política de Calidad de la Facultad, la cual incluye los 5 objetivos de calidad que son:

- Elevar la calidad de la formación integral universitaria, tanto de sus estudiantes, profesores como del personal administrativo.

- Contribuir al desarrollo de la investigación socialmente pertinente a través de la formación y la consolidación de grupos y líneas de investigación que permitan la generación, aplicación y transmisión de conocimientos, así como productos científicos enfocados al desarrollo humano sostenible, congruentes con la agenda de desarrollo local y nacional.

- Aumentar la extensión y vinculación con el entorno mediante la participación comprometida de la Facultad, generando y apoyando proyectos a través de alianzas estratégicas con los diferentes actores sociales, en beneficio de la sociedad, particularmente con la industria.

- Mejorar la práctica de una gestión ética y de calidad, la toma de decisiones basadas en el marco normativo vigente, asegurando un adecuado clima laboral, desarrollando talentos en el recurso humano, manteniendo y mejorando la infraestructura, con procesos internos altamente participativos $e$ incluyentes, con especial énfasis en materia de transparencia.

- Contribuir al desarrollo de las buenas prácticas en materia de Seguridad y Salud en el trabajo, previniendo lesiones y enfermedades; así como fomentar el cuidado del Medio Ambiente en el desarrollo de las actividades de la comunidad de la Facultad.

\section{Proyecto}

Se realiza con la misma estructura con la que se lleva a cabo el proyecto de Planeación Táctica del Cuerpo Académico: Objetivo General, Objetivos Específicos, Metas, Estrategias, Acciones y Recursos. 


\section{Cronograma}

Se planifican las acciones a realizar mensualmente durante el año de análisis. Este apartado facilita la visualización de las acciones propuestas a desarrollar alineadas a los objetivos de calidad de la Política de Calidad, así como a los Programas Prioritarios y las Estrategias establecidas en el Plan de Desarrollo.

\section{Desarrollo en prosa}

$\mathrm{Y}$, por último, se muestra el proyecto de Planeación Operativa con la estructura de un proyecto de investigación científica a fin de puntualizar los detalles. La estructura requerida para el desarrollo en prosa del proyecto es: Introducción, Justificación, Metodología, Resultados esperados, Conclusiones y Bibliografía

\section{Resultados}

Considerando la visión 2020, la Facultad ha establecido metas anuales en porcentaje de Cuerpos Académicos Consolidados, buscando incrementar un $10 \%$ durante un periodo de 12 años. Actualmente se cuentan con $14 \mathrm{CA}$ Consolidados, es decir, un 38\% del total de CA. Comparando con los resultados obtenidos durante el año 2018, se logró aumentar un 4.7\% en el 2019 y estar dentro de la meta visualizada.

A su vez, también se ha considerado una tendencia de metas anuales para los CA en Proceso de Consolidación. En el transcurso del año 2019 se redujo un $0.25 \%$ el porcentaje de Cuerpos Académicos en Proceso de Consolidación de acuerdo al total de CA registrados, es decir, contar con un 30\%.

Para lograr una mejor visibilidad, se ha realizado un análisis al tomar el porcentaje total que están abarcando tanto los Cuerpos Académicos en Proceso de Consolidación como los Cuerpos Académicos ya consolidados en comparación con el total de los 37 CA. Ambos formando un $68 \%$ de este total.

Resultado obtenido del periodo - CA en Consolidación y Consolidados.

\section{Evolución de Cuerpos Académicos de la FIME}

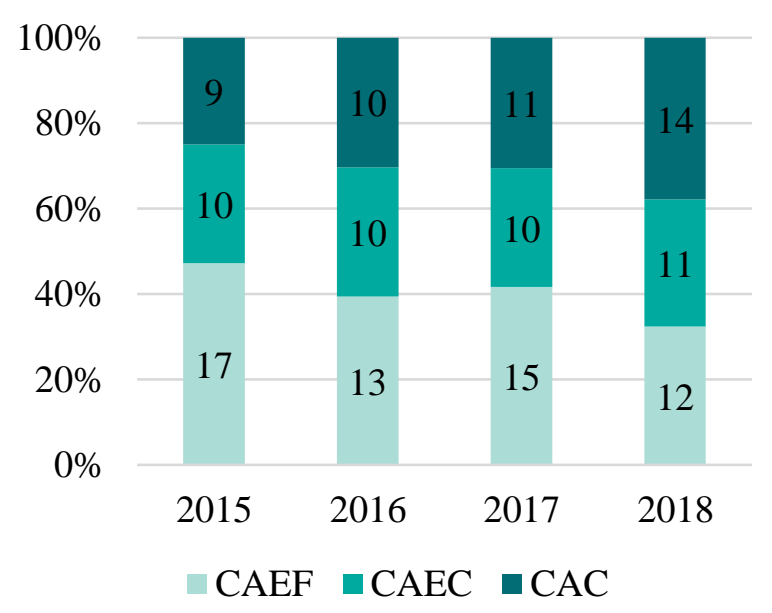

Gráfico 1 Evolución de Cuerpos Académicos de la FIME

\section{Porcentaje de Cuerpos Académicos de la Facultad}

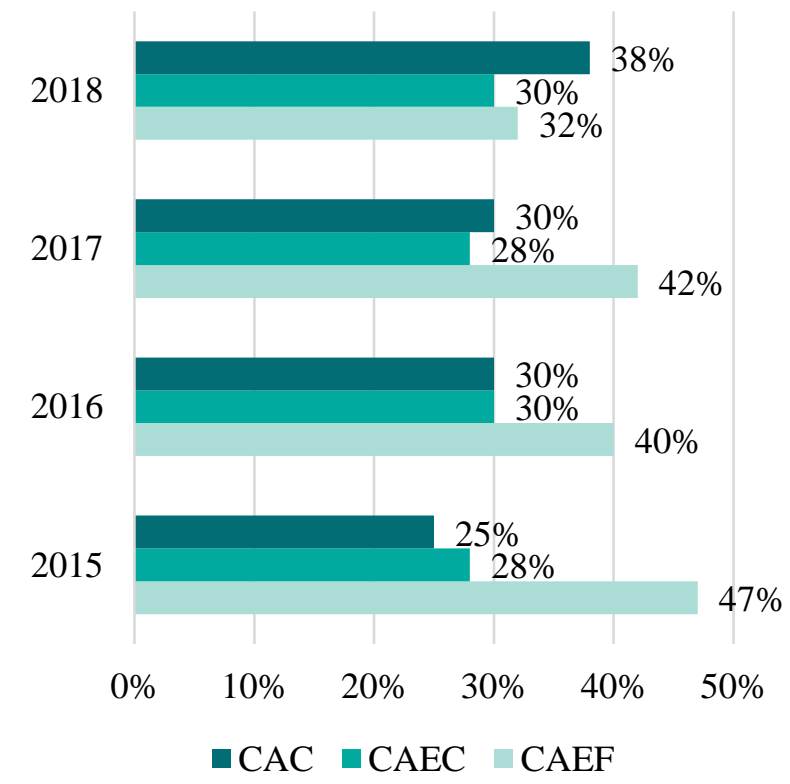

Gráfico 2 Porcentaje de Cuerpos Académicos de la Facultad

\section{Agradecimientos}

Se agradece la colaboración de la Coordinación de Capacidad Académica y Asesorías Académicas al facilitar los datos estadísticos en relación a la evolución de los cuerpos académicos. 


\section{Conclusión}

El fomentar la cultura de planeación de los Cuerpos Académicos ha permitido desarrollar un trabajo colegiado con dirección e integración entre sus miembros tributando al fortalecimiento de los programas educativos del área de ingeniería. Para asegurar la transición de los Cuerpos Académicos es importante considerar los criterios de evaluación que promueve el PRODEP para realizar un análisis de la situación actual del cuerpo que permita definir líneas de acción estratégicas a fin de integrar el plan de mejora. Para que un Cuerpo Académico se vaya fortaleciendo es necesario que todos los miembros tengan líneas de generación y aplicación del conocimiento definido y trabajen en conjunto $y$ en colaboración con otros Cuerpos Académicos para cultivarlas. El trabajo que realiza estos grupos de profesores es integral pues permite el desarrollo de profesores y también contribuye en la formación de los estudiantes ya que su participación en trabajos investigativos conlleva el desarrollo de competencias que se reflejarán en el fortalecimiento de su perfil en beneficio de la sociedad, al generar profesionistas que aporten al conocimiento y contribuyan al desarrollo laboral, económico y social. La planificación del trabajo colegiado aparte de tributar al fortalecimiento de la planta académica impacta positivamente en la consolidación de los programas académicos de la institución. La realización de este ejercicio facilita analizar los criterios de evaluación de los organismos que son referente en este contexto, afín de alinear las prácticas realizadas a los estándares de calidad que se evalúan en la categoría de docentes en el campo de la educación superior.

\section{Referencias}

Facultad de Ingeniería Mecánica y Eléctrica. (2012). Plan de Desarrollo. Obtenido de Facultad de Ingeniería Mecánica y Eléctrica: http://www.fime.uanl.mx/Plan\%20de\%20desarr ollo\%20FIME\%20Vision\%202020.pdf

Chiavenato, I. (2016). Planeación Estratégica. Río de Janeiro: Mc Graw Hill Education.

Facultad de Ingeniería Mecánica y Eléctrica. (24 de Septiembre de 2018). Política de Calidad. Obtenido de Facultad de Ingeniería Mecánica y Eléctrica: http://www.fime.uanl.mx/politicas.html

ISSN-2523-2460

ECORFAN $^{\circledR}$ Todos los derechos reservados
Gutiérrez Sáenz, R., \& Sánchez González, J. (1990). Metodología del trabajo intelectual. México: Esfinge.

Liniers, M. C. (2004). El Análisis Documental: Indización y Resumen. CINDOC-CSIC.

Maya, E. (2014). Métodos y técnicas de Investigación Una propuesta Una propuesta ágil para la presentación de trabajos científicos en las áreas de arquitectura, urbanismo y disciplinas afines. Obtenido de Arquitectura UNAM:

https://arquitectura.unam.mx/uploads/8/1/1/0/8 110907/metodos_y_tecnicas.pdf

Münch Galindo, L. (2008). Planeación Estratégica: El rumbo hacia el éxito. Trillas.

Ramos, G. (2019). Promover la calidad, equidad y relevancia del sistema de educación superior en México. Ciudad de México: OECD.

Secretaría de Educación Pública. (7 de Febrero de 2019). Reglas de operación del programa para el desarrollo profesional docente para el ejercicio fiscal 2019. Obtenido de Diario Oficial de la Federación: https://dof.gob.mx/nota_detalle.php?codigo $=55$ $51600 \&$ fecha $=28 / 02 / 2019$

Secretaría de Educación Pública. (28 de Febrero de 2019). Reglas de Operación del Programa para el Desarrollo Profesional Docente para el ejercicio fiscal 2019,. Obtenido de Diario Oficial de la Federación: http://dof.gob.mx/DOFmobile/nota_detalle_pop up.php?codigo $=5552808$

Secretaría de Educación Pública. (s.f.). Objetivos Dirección de Superación Académica. Obtenido de Dirección General de Educación Superior Universitaria : Dirección General de Educación Superior Universitaria

UNESCO. (2016). Educación 2030: Declaración de Incheon y Marco de Acción para la realización del Objetivo de Desarrollo Sostenible 4: Garantizar une aducación inclusiva y equitativa de calidad y promover oportunidades de aprendizaje permanente para todos. Obtenido de UNESDOC: https://unesdoc.unesco.org/ark:/48223/pf00002 45656_spa/PDF/245656spa.pdf.multi

OCHOA-VARGAS, Margarita \& CHÁVEZ-LÓPEZ, María Margarita. Telesecundaria: entre la teoría y la práctica. Revista de Educación Técnica. 2019 